\title{
Factors Related to the Spontaneous Regression of Cancer
}

\author{
"Spontaneous regression of cancer is the most fascinating chapter of medicine" Willam Osler
}

\section{Editorial}

D.S. Botseas

Received 21/05/2012 Accepted 30/06/2012

\begin{abstract}
The goal of the present editorial is to remind us of a very rare phenomenon in medicine, which is the spontaneous regression of cancer.

Factors related to this type of regression are mentioned. Among them are the following:

- Infections

- Fever and therapeutic hyperthermia

- Hormonal factors

- Various other factors

- Exclusion of inaccurate diagnosis of malignancy of a tumour is essential to the validity of reported cases.
\end{abstract}

Key words:

Cancer, Spontaneous regression, Regression of cancer

\section{Introduction}

Spontaneous regression of cancer is a rare phenomenon that occurs once in every 60.000 to 100.000 cancer victims [1,2]. In some particular types of cancer, it is more frequent. For example, in melanoma it is estimated to happen in one out of 400 cases of melanoma patients. Likewise, in infant neuroblastoma, it occurs in one out of 6 patients with this particular cancer. Infants under one year of age, in particular, have a very good chance of undergoing spontaneous regression in contrast to older children, among whom this cancer may behave rather aggressively [3].

Several investigators have discovered a number of factors related to spontaneous regression of cancer. Some of these factors are listed below.

\section{Factors Related to the Spontaneous Regres- sion of Cancer}

- Infections or suppuration of wounds is a factor which has been correlated with spontaneous regression in

D.S. Botseas M.D., F.A.C.S. (Corresponding author) - Surgeon Athens General Clinic \& Hygeia Hospital, Athens, Greece - F. resident Roswell Park Cancer Center, Buffalo N.York $\square$ e-mail: typogramma@gmail.com cancer patients. In 1868, professor Busch [4] reported a cancer patient whose tumour regressed following an infection by Streptococcus pyogenes. This particular case, possibly, inspired Coley [5-7], a surgeon at Memorial Hospital in New York, to prepare an extract from killed Streptococcus pyogenes bacteria, which he used to inject into the tumours of patients suffering from advanced sarcomas that were considered to be inoperable. The injections were administered twice a week and for periods extending to several months. This method, however, was overshadowed by the progress of radiotherapy and chemotherapy. It was eventually forgotten after Coley's death in 1936.

Recent studies, however, by Wiemann and Starnes (1994) [8] revealed that advanced sarcoma patients who had received such injections had a 5-year survivial of $44 \%$. In addition to this finding, other studies by Nauts and McLaren [9] revealed that those batches of Collin's "toxin" - as the aforementioned preparation was known - that produced high fever, were more effective than other series. Administered over 6 months, these specific infections achieved an increased patient survival of $80 \%$ that lasted from 5 to 88 years.

Another report by Ruckdeschel [10] demonstrated that patients who developed empyema after pulmonary surgery for lung carcinoma increased their survival by $50 \%$.

Likewise, Hoption Cann et al [11] reported the case of a patient with an inoperable, advanced carcinoma of the pancreas who developed peritonitis, following which his cancer disappeared. They also found 3 similar cases in the literature, two of whom had also presented with high fever. A similar case of inoperable carcinoma of the stomach is reported by Army Surgeon Kousouris [12], who decided to perform a palliative gastroenterostomy. Postoperatively, the patient developed peritonitis, due to a small leak of the anastomosis. He recovered after conservative therapy. Strangely enough, the cancer disappeared postoperatively.

- Fever is frequently related to the spontaneous regression of cancer, and is considered to be part of the body response to infection. According to Hobohm 
$[13,14]$, cancer cells are less resistant to fever than normal cells. The remains of necrotic cancer cells provide tumour antigens, which help dendritic cells (D.C.) to mature [15], thus energizing T-lymphocytes to act immunologically. Similarly, heat-therapy or hyperthermia ( $41^{\circ} \mathrm{C}$ for 6 hours) mobilizes the actions of cytokines (IL1, IL6, INF, CNTF, INFA) in a therapeutic way.

- Another factor is related to hormonal influences. The effect of hormones on the growth of cancer of the breast and the prostate is well known. A very impressive case of cancer growth related to the thyroid hormone is reported by Hercbergs [16]. It concerns a patient with carcinoma of the lung, who also developed hypothyroid coma. When he finally recovered from the coma, his carcinoma had disappeared.

- Other factors related to the spontaneous regression of cancer are:

a) the interruption of carcinoma promoters (carcinogens) as in some cases of ureterosigmoidostomy, which stops the flow of urine (that possibly contains carcinogens) from the urinary bladder;

b) allergy;

c) unusual sensitivity of cancers cells to therapies that are considered inadequate;

d) damage to the vascular supply of the tumour during an inadequate excision;

e) psychological and other factors that current knowledge has not yet understood. W.H. Cole [17] summarizes these factors as being numerous, and they include bacterial products, enzymes, infections, hormones, trauma etc.

The accuracy of the histologic diagnosis The highly laborious research of Everson [18,19] in verifying the histologic diagnosis of the 1000 cases of spontaneous regression of cancer reported in the literature ran into many difficulties during his attempt to locate the histology slides and have them reevaluated by two other pathologists. Eventually, they were able to verify that 130 of the patients were suffering from cancer.

His reason for getting involved in such an undertaking was because occasionally a histologic diagnosis of cancer may subsequently emerge as a benign tumour, following examination by another pathologist. Likewise, widespread nodules in the lungs of a patient considered to be metastatic spread, may be due to some other cause. Even a case of multiple pulmonary lesions due to many small infarcts has been reported.

\section{Conflict of interest}

The authors declare that they have no conflict of interest.

\section{SpringerWien NewYork}

\section{References}

1. Black, M. et al.: "Structural presentation of Tumor-host relationships in gastric cancer". Surg. Gyn Obst. 1956, 159: 1059-61.

2. Boyd W.: "Spontaneous Regression of Cancer Springfield" C.C. Thomas, 1966

3. Koop C.E. et al.: "Neuroblastoma in Childhood Surgery" 1955: Surgery 38: 272 .

4. Busch W (1867). “Aus dersitzung der medicinichen.”. Berliner Klinische Wochenschrift 5: 137.

5. Coley WB (1893). "The Treatment of Malignant Tumors by Repeated Innoculations of Erysipelas: With a Report of Ten Original Cases.". American Journal of the Medical Sciences 10: 487-511.

6. Coley WB. Contribution of the knowledge of sarcoma. Annals of Surgery 1891

7. Edward F McCarthy.The toxins of William B. Coley and the treatment of bone and soft-tissue sarcomas Iowa Orthop J. 2006; 26: 154-158.

8. Wiemann B, Starnes CO. Coley's toxins, tumor necrosis factor and cancer research: a historical perspective. Pharmacol Ther. 1994;64(3):529-64.

9. Nauts HC, McLaren JR. Coley toxins--the first century. Adv Exp Med Biol. 1990;267:483-500.

10. Ruckdeschel JC, Codish SD, Stranahan A, McKneally MF. Postoperative empyema improves survival in lung cancer. Documentation and analysis of a natural experiment. N Engl J Med.1972 Nov 16;287(20):1013-1017

11. Hoption Cann et al.: "Spontaneous regression of pancreatic cancer" Case Rep. Cl. Rev. 2004, 693-6.

12. Kousouris P.: Personal communication.

13. Hobohm U.: "Pathogen associated molecular pattern in cancer, immunotherapy". 2008, 128(2): 95-107.

14. Hobohm U.: "Fever therapy revisited" Br. J. Cancer 2005, 92(3) 421-425.

15. Basu S., Srivastava R.: "Fever like temperature induces maturation of dendritic-cells through induction of HSP. 90. Int. Immunol. 2003; 15. 9: 1053-61 [Pub. Med.]

16. Hercbergs A. Spontaneous remission of cancer--a thyroid hormone dependent phenomenon? Anticancer Res. 1999 NovDec;19(6A):4839-44. Review

17. Cole W.: "Efforts to explain spontaneous regression of cancer" J. Surg. Oncol. 1981; 17(3): 201-209 [Pub, Med]

18. Everson T.C.: "Spontaneous regression of cancer: primary report" Ann of Surg. 1956, 144(3): 366-381.

19. Everson T.C.: "Spontaneous regression of cancer". Annals of N.Y. Academy of Sciences. 1964, 114: 721-736. 